Article

\title{
Silica Sulfuric Acid Promotes Aza-Michael Addition Reactions under Solvent-Free Condition as a Heterogeneous and Reusable Catalyst
}

\author{
Yan Wang, Yan-Qin Yuan and Sheng-Rong Guo * \\ Department of Chemistry and Biology, Lishui University, Lishui, Zhejiang 323000, China \\ * Author to whom correspondence should be addressed; E-Mail: guosr9608@163.com.
}

Received: 3 November 2009; in revised form: 19 November 2009 / Accepted: 23 November 2009 / Published: 23 November 2009

\begin{abstract}
A highly efficient, inexpensive, recyclable, convenient, and green protocol for chemoselective aza-Michael addition reactions of amines/thiols to $\alpha, \beta$-unsaturated compounds using silica sulfuric acid $\left(\mathrm{SSA}\right.$ or $\left.\mathrm{SiO}_{2}-\mathrm{SO}_{3} \mathrm{H}\right)$ was developed. This method is simple, convenient and the title compounds are produced in good to excellent yields.
\end{abstract}

Keywords: amines; thiols; silica sulfuric acid (SSA); Michael reaction; $\alpha, \beta$-unsaturated olefins

\section{Introduction}

The Michael reaction has been studied for over a century. The conjugate addition of amines to carbon-carbon double bonds is a useful protocol in synthetic organic chemistry [1-4]. It is used extensively in the synthesis of pharmaceutical intermediates, peptide analogues, antibiotics, and other biologically active molecules and drugs [5-10]. In the past few years, a number of alternative procedures have been developed for the conjugate addition of amines to $\alpha, \beta$-unsaturated carbonyl compounds. In particular, various Lewis acid catalyzed reactions have been reported. This reaction has been investigated using catalysts such as lanthanum trichloride $\left(\mathrm{LaCl}_{3}\right)$ [11], bromodimethylsulfonium bromide [12], silica supported perchloric acid [13], cerium(IV) ammonium nitrate (CAN) [14,15], $\beta$-cyclodextrin [16], zirconium(IV) chloride [17], samarium(III) triflate [18], $\mathrm{ZrOCl}_{2} \cdot 8 \mathrm{H}_{2} \mathrm{O}$ on montmorillonite $\mathrm{K}_{10}$ [19], $\mathrm{N}$-methylimidazole [20], $\mathrm{Cu}(\mathrm{acac})_{2}$ /ionic liquid [21], 1,8-diazabicyclo[5.4.0] 


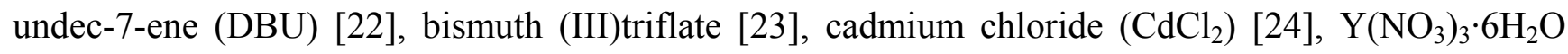
[25], and cellulose supported copper(0) [26]. Unfortunately, many of these processes suffer from limitations, such as the use of expensive reagents, harsh conditions, relatively long reaction times, high catalyst loading, low selectivity and tedious work-up procedures for their separation, recycling, or disposal problems and effluent pollution. All these limitations prompted us to explore other better catalysts, which have more efficiency and less limitations. We tried to find a catalyst that has certain properties, such as the good thermal and mechanical stabilities of supported reagents, is easy to handle, of low toxicity, non-corrosive, easy to separate from reaction mixtures through filtration, and feasible for reuse.

Very recently, silica sulfuric acid (SSA) has been widely used as a reusable, heterogeneous, inexpensive solid Brønsted acid catalyst and has received much attention [27-30] and there has been increasingly awareness about the use of solid acids such as silica sulfuric acid (SSA) for synthesizing organic intermediates and fine chemicals [31-34]. SSA is a strong Brønsted and Lewis acid, presumably arising from the formation of $\mathrm{SiO}_{2}-\mathrm{SO}_{3} \mathrm{H}$ sites on the surface. This heterogeneous catalyst can be easily separated from the reaction media, has greater selectivity, and is recyclable, easier to handle, more stable, nontoxic, and insoluble in organic solvents. We hoped that SSA would be a superior proton source to the standard acidic solid supports for running Michael reactions under heterogeneous conditions, and herein we wish to report the use of SSA as a reusable solid acid catalyst for the Michael addition reaction.

\section{Results and Discussion}

In a set of initial experiments, acrylic acid 2-phenylsulfanyl-ethyl ester(PTEA)was allowed to react with morpholine in the presence of a varying quantities of $\mathrm{SiO}_{2}-\mathrm{SO}_{3} \mathrm{H}$. The results show that an excellent yield of the Michael adduct can be achieved by reacting a mixture of morpholine (1.2 equiv.) and PTEA (1.0 equiv) in the presence of $\mathrm{SiO}_{2}-\mathrm{SO}_{3} \mathrm{H}(\mathrm{SSA}, 100 \mathrm{mg}$ ) at room temperature (Scheme 1). This encouraged us to exploit the generality and scope of this reaction catalyzed by $\mathrm{SiO}_{2}-\mathrm{SO}_{3} \mathrm{H}$ by using other Michael acceptors with various aromatic and aliphatic amines. As shown in Table 1, the Michael addition of various aliphatic amines and aryl amines carrying either electron-donating or electron-withdrawing groups were successfully reacted with PTEA or PEEA to produce their corresponding Michael adducts in high to excellent yields. The addition of primary amines such as benzylamine resulted in only monoalkylated products; the aromatic amines showed poor reactivity compared to the aliphatic amines. No side products were observed when using excess amines; pure products could be obtained by removal of the catalyst by filtration followed by column chromatography. The Michael addition of heteroaromatic imidazole with PTEA took more time (analysis by TLC and GC) as compared with other amines and gave a low yield of product which may be due to the weak nucleophilicity of the imidazole molecule steming from its aromaticity. The reactions with 4-nitroaniline and 2-aminophenol did not proceed at all, even at higher temperatures (entries 13, 14). 
Scheme 1. Michael addition of aliphatic and aromatic amines with PTEA or PEEA.

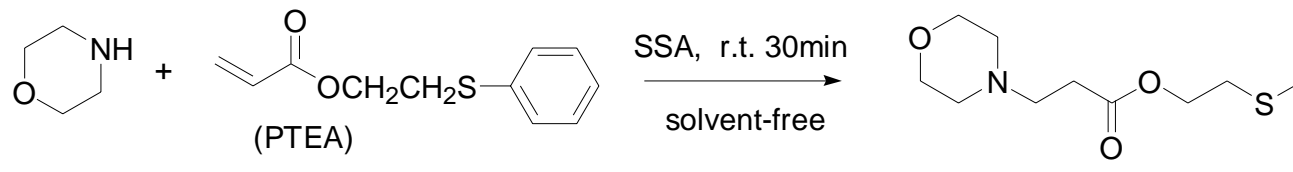

$\mathrm{R}+\mathrm{N}_{\mathrm{OCH}_{2} \mathrm{CH}_{2} \mathrm{~S}}^{\mathrm{NH}}+\underset{\text { solvent-free }}{\mathrm{SSA}, 60{ }^{\circ} \mathrm{C}}$

(PEEA)

Table 1. Michael addition of amines catalyzed by SSA under solvent-free conditions.

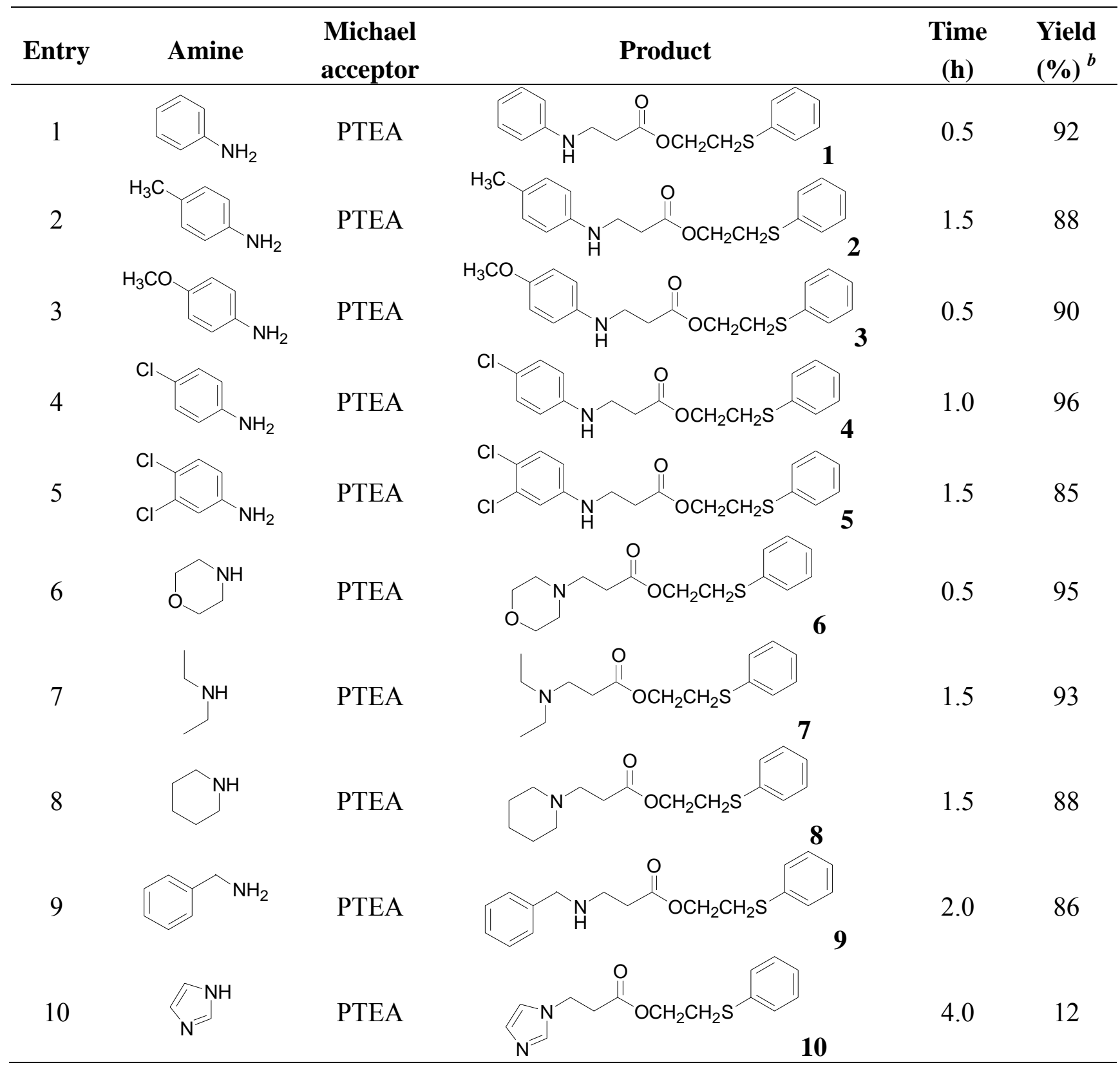


Table 1. Cont.

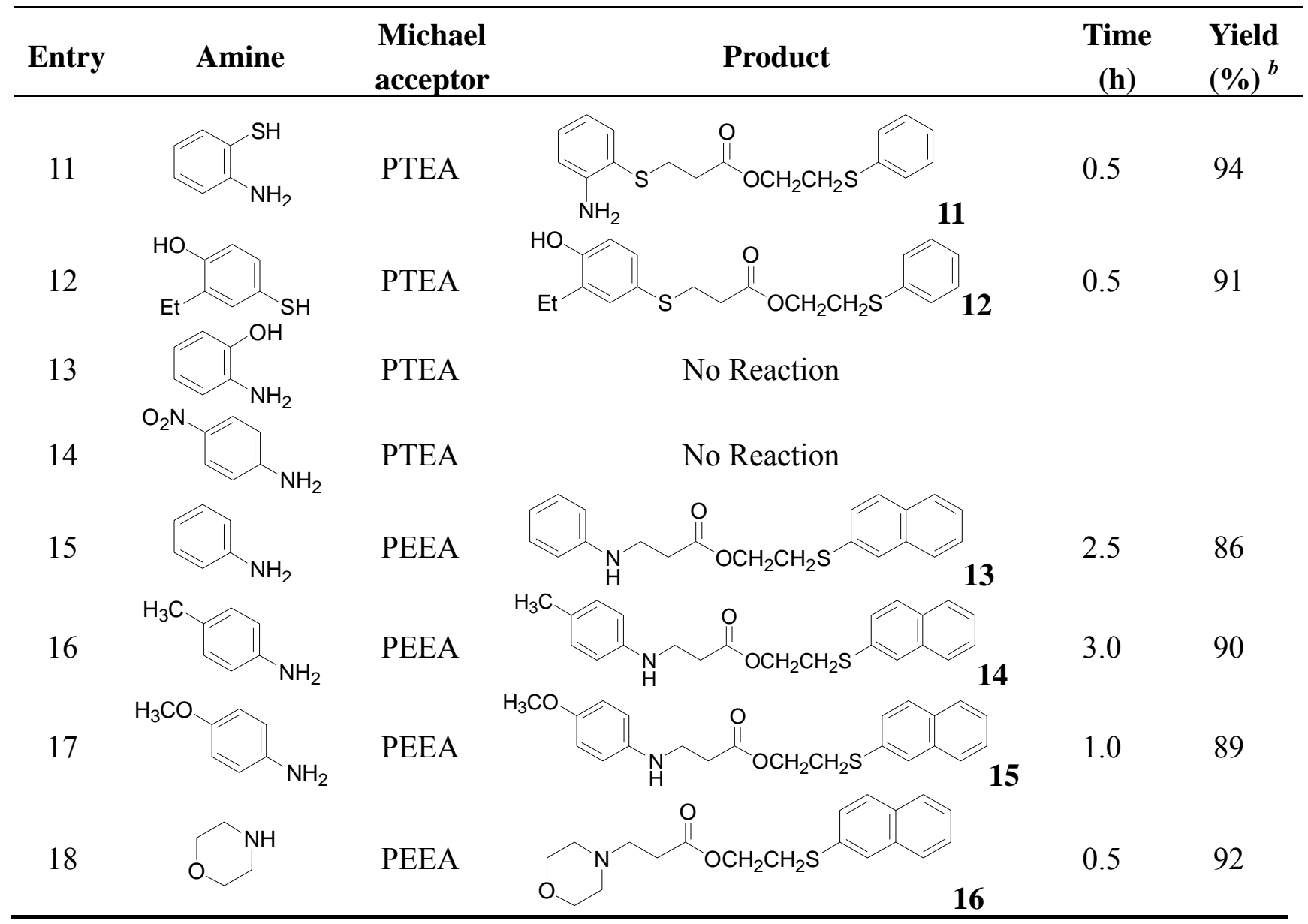

An experiment was performed to show the chemoselectivity between thiols and aniline by using 2-aminobenzenethiol $(1.2 \mathrm{mmol})$ and PTEA $(1.0 \mathrm{mmol})$ in the presence of SSA $(0.1 \mathrm{~g})$ at room temperature. After $30 \mathrm{~min}$, compound $\mathbf{1 1}$ was the only product (Scheme 2).

Scheme 2. Michael addition of 2-aminobenzenethiol to PTEA.

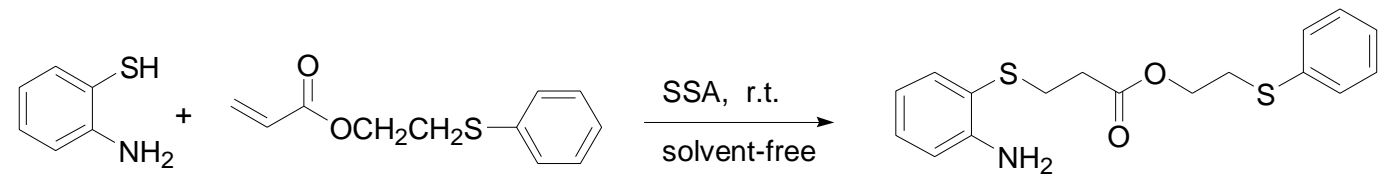

Another competition reaction was also carried out by using 3-ethyl-4- hydroxythiophenol (1.2 mmol), and PTEA $(1 \mathrm{mmol})$ in the presence of SSA $(0.1 \mathrm{~g})$ at room temperature, and compound 12 was formed as the only product (Scheme 3). From the above two experiments, we conclude that the activity of the amines and phenols are both less than that of thiols. 
Scheme 3. Michael addition of 3-ethyl-4-hydroxythiophenol to PTEA.

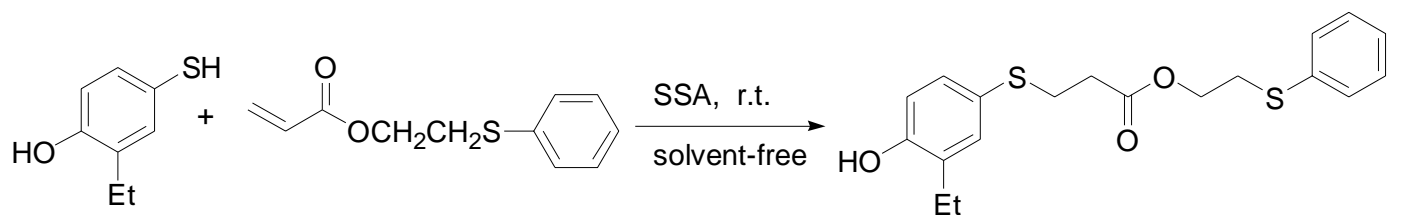

We also examined the reaction of aniline with PTEA using different protic and aprotic solvents in the presence of $\mathrm{SiO}_{2}-\mathrm{SO}_{3} \mathrm{H}$ at different temperature. The results showed that solvent-free conditions gave a better yield of $\beta$-amino compound (Table 2 ).

Table 2. Michael addition reaction of PTEA with aniline in protic and aprotic solvents.

\begin{tabular}{ccc}
\hline Entry & Solvent & Yield (\%) \\
\hline 1 & $\mathrm{C}_{2} \mathrm{H}_{5} \mathrm{OH}$ & 72 \\
2 & $\mathrm{H}_{2} \mathrm{O}$ & 34 \\
3 & $\mathrm{CH}_{3} \mathrm{CO}_{2} \mathrm{H}$ & 78 \\
4 & $\mathrm{ClCH}_{2} \mathrm{CH}_{2} \mathrm{Cl}$ & 83 \\
5 & $\mathrm{Toluene}$ & 41 \\
6 & $\mathrm{CH}_{3} \mathrm{CN}$ & 86 \\
7 & $\mathrm{CH}_{2} \mathrm{Cl}_{2}$ & 86 \\
8 & $\mathrm{Neat}$ & 91 \\
\hline
\end{tabular}

The catalyst system was found to be recyclable and the reaction condition can be scaled up. In order to test the reusability of the catalyst, a reaction of PTEA $(10 \mathrm{mmol})$ and morpholine $(12 \mathrm{mmol})$ was carried out in the presence of SSA $(1.0 \mathrm{~g})$ and the catalyst was recovered after completion and activated by heating at $100{ }^{\circ} \mathrm{C}$ under vacuum for $1 \mathrm{~h}$. The recovered catalyst was reused for the aza-Michael reaction of another batch of PTEA $(10 \mathrm{mmol})$ and morpholine $(12 \mathrm{mmol})$ giving 90\% yield of the desired product after $1 \mathrm{~h}$. Again, the catalyst was recovered, reactivated and reused repeatedly for three more consecutive times for aza-Michael reactions affording $85 \%, 70 \%$ and $70 \%$ yields, respectively. From this observation, it is clear that the reaction can be scaled up and the catalyst is reusable with a slight decrease in catalytic activity.

To compare the catalytic activity of $\mathrm{SiO}_{2}-\mathrm{SO}_{3} \mathrm{H}$ with ordinary silica, an identical experiment was performed by stirring an intimate mixture of PTEA $(1.0 \mathrm{mmol})$, morpholine $(1.2 \mathrm{mmol})$ and silica (240-400 mesh, $100 \mathrm{mg}$ ) at room temperature. No aza-Michael addition product was formed even after $24 \mathrm{~h}$, which indicate that the requirement of $\mathrm{SiO}_{2}-\mathrm{SO}_{3} \mathrm{H}$ is key factor for the successful outcome of the reaction. Furthermore, using of $\mathrm{SiO}_{2}-\mathrm{SO}_{3} \mathrm{H}$ in the aza-Michael addition reduces the cost of the catalysts, and the reaction completed in less time. It is not very clear whether $\mathrm{SiO}_{2}-\mathrm{SO}_{3} \mathrm{H}$ act as a solid acid catalyst or as a reservoir providing a small concentration of acid in solution. However, from the experiments carried out using recovered $\mathrm{SiO}_{2}-\mathrm{SO}_{3} \mathrm{H}$ suggest that it acts as a solid acid in the aza-Michael reactions. 


\section{Experimental}

\section{General}

All reagents were purchased from commercial sources and used without further purification. TLC analysis was performed with glass backed plates precoated with silica gel and examined under UV (254 nm). NMR spectra were measured in $\mathrm{CDCl}_{3}$ with $\mathrm{Me}_{4} \mathrm{Si}$ as the internal standard on a Bruker Advance 300 instrument at room temperature. IR spectra were recorded on Bruker FT-IR spectrometer, absorbances are reported in $\mathrm{cm}^{-1}$. Elemental analyses were performed on a Perkin-Elmer-2400 elemental analyzer.

\section{General procedure for the synthesis of $\beta$-amino carbonyl compounds}

A mixture of aniline $(1.2 \mathrm{mmol})$ and Michael acceptor PTEA (1.0 mmol or PEEA $1.0 \mathrm{mmol})$ and SSA $(0.1 \mathrm{~g})$ was stirred at room temperature for $0.5-6 \mathrm{~h}$. The reaction was monitored by TLC. Upon completion, EtOAc $(5 \mathrm{~mL})$ was added and the $\mathrm{SiO}_{2}-\mathrm{SO}_{3} \mathrm{H}$ catalyst can be reused after it was removed by filtration. The filtrate was washed with saturated $\mathrm{NaHCO}_{3}(\mathrm{aq})$ and brine, then dried with anhydrous $\mathrm{Na}_{2} \mathrm{SO}_{4}$, and concentrated under vacuum. The residue was analyzed by NMR. In most cases, pure product was obtained. If needed, the residue was further purified by silica gel column chromatography using a 4:1 hexane-EtOAc mixture as eluent to afford the pure product. All of the products are new compounds, and the structures of products were determined from their NMR, MS or elemental analyses.

\section{Selected spectroscopic data}

3-Phenylaminopropionic acid 2-phenylsulfanyl-ethyl ester (1): yellow oil; ${ }^{1} \mathrm{H}-\mathrm{NMR} \delta$ : 7.42 (d, $J=7.7 \mathrm{~Hz}, 2 \mathrm{H}), 7.18-7.34(\mathrm{~m}, 5 \mathrm{H}), 6.75(\mathrm{t}, J=7.3 \mathrm{~Hz}, 1 \mathrm{H}), 6.62(\mathrm{~d}, J=8.0 \mathrm{~Hz}, 2 \mathrm{H}), 4.30(\mathrm{t}$, $J=6.8 \mathrm{~Hz}, 2 \mathrm{H}), 3.95($ br $1 \mathrm{H}), 3.46(\mathrm{t}, J=6.3 \mathrm{~Hz}, 2 \mathrm{H}), 3.17(\mathrm{t}, J=6.8 \mathrm{~Hz}, 2 \mathrm{H}), 2.62(\mathrm{t}, J=6.3 \mathrm{~Hz}, 2 \mathrm{H})$; ${ }^{13} \mathrm{C}-\mathrm{NMR} \delta$ : 172.1, 147.5, 134.9, 130.1, 129.3, 129.1, 126.7, 117.8, 113.1, 63.0, 39.4, 33.9, 32.5; Anal. Calcd. for $\mathrm{C}_{17} \mathrm{H}_{19} \mathrm{NO}_{2} \mathrm{~S}$ : C, 67.74; H, 6.35. Found: C, 67.76; H, 6.34.

3-p-Tolylaminopropionic acid 2-phenylsulfanyl-ethyl ester (2): yellow oil; ${ }^{1} \mathrm{H}-\mathrm{NMR} \delta$ : 7.43-7.21 (m, $5 \mathrm{H}), 7.02(\mathrm{~d}, J=8.2 \mathrm{~Hz}, 2 \mathrm{H}), 6.58$ (d, $J=8.2 \mathrm{~Hz}, 2 \mathrm{H}), 4.29$ (t, $J=6.8 \mathrm{~Hz}, 2 \mathrm{H}), 3.93(\mathrm{br}, 1 \mathrm{H}), 3.44$ (t, $J=6.3 \mathrm{~Hz}, 2 \mathrm{H}), 3.16(\mathrm{t}, J=6.8 \mathrm{~Hz}, 2 \mathrm{H}), 2.60(\mathrm{t}, J=6.2 \mathrm{~Hz}, 2 \mathrm{H}), 2.27(\mathrm{~s}, 3 \mathrm{H}) ;{ }^{13} \mathrm{C}-\mathrm{NMR} \delta: 172.2$, 145.2, 134.9, 130.1, 129.8, 129.1, 127.0, 126.7, 113.3, 62.9, 39.8, 33.8, 32.5, 20.4; Anal. Calcd. for $\mathrm{C}_{18} \mathrm{H}_{21} \mathrm{NO}_{2} \mathrm{~S}: \mathrm{C}, 68.54 ; \mathrm{H}, 6.71$. Found: $\mathrm{C}, 68.56 ; \mathrm{H}, 6.68$.

3-(4-Methoxyphenylamino)-propionic acid 2-phenylsulfanyl-ethyl ester (3): Off-white oil; ${ }^{1} \mathrm{H}-\mathrm{NMR} \delta$ : 7.23-7.42 (m, 5H), $6.80(\mathrm{q}, J=2.3 \mathrm{~Hz}, J=6.9 \mathrm{~Hz}, 2 \mathrm{H}), 6.62(\mathrm{~d}, J=2.3 \mathrm{~Hz}, J=6.9 \mathrm{~Hz}, 2 \mathrm{H}), 4.29$ (t, $J=6.8 \mathrm{~Hz}, 2 \mathrm{H}), 3.76(\mathrm{~s}, 3 \mathrm{H}), 3.43(\mathrm{br}, 1 \mathrm{H}), 3.40(\mathrm{t}, J=6.3 \mathrm{~Hz}, 2 \mathrm{H}), 3.16(\mathrm{t}, J=6.8 \mathrm{~Hz}, 2 \mathrm{H}), 2.58$ (t, $J=6.3 \mathrm{~Hz}, 2 \mathrm{H}) ;{ }^{13} \mathrm{C}-\mathrm{NMR} \delta: 172.2,152.5,141.7,135.0,129.8,129.0,126.7,114.9,114.6,63.9,55.8$, 40.5, 33.9, 32.5; Anal. Calcd. for $\mathrm{C}_{18} \mathrm{H}_{21} \mathrm{NO}_{3} \mathrm{~S}$ : C, 65.23; H, 6.39. Found: C, 65.15; H, 6.34. 
3-(4-Chlorophenylamino)propionic acid 2-phenylsulfanyl-ethyl ester (4): Off-white oil; ${ }^{1} \mathrm{H}-\mathrm{NMR} \delta$ : $7.41(\mathrm{~d}, J=1.5 \mathrm{~Hz}, 2 \mathrm{H}), 7.23-7.39(\mathrm{~m}, J=6.4 \mathrm{~Hz}, 3 \mathrm{H}), 7.13(\mathrm{t}, J=2.2 \mathrm{~Hz}, 2 \mathrm{H}), 6.59$ (t, $J=2.0 \mathrm{~Hz}$, 2H), $4.29(\mathrm{t}, J=6.8 \mathrm{~Hz}, 2 \mathrm{H}), 3.97(\mathrm{br}, 1 \mathrm{H}), 3.42(\mathrm{t}, J=6.2 \mathrm{~Hz}, 2 \mathrm{H}), 3.16(\mathrm{t}, J=6.8 \mathrm{~Hz}, 2 \mathrm{H}), 2.58$ (t, $J=6.2,2 \mathrm{H}) ;{ }^{13} \mathrm{C}-\mathrm{NMR} \delta: 171.0,143.9,136.4,129.6,129.3,128.9,126.1,125.1,114.9,63.6,39.0$, 37.8, 35.7; Anal. Calcd. for $\mathrm{C}_{17} \mathrm{H}_{18} \mathrm{ClNO}_{2} \mathrm{~S}: \mathrm{C}, 60.80 ; \mathrm{H}, 5.40$. Found: C, 60.83; H, 5.42.

3-(3,4-Dichlorophenylamino)propionic acid 2-phenylsulfanyl-ethyl ester (5): yellow oil; ${ }^{1} \mathrm{H}-\mathrm{NMR} \delta$ : 7.56-7.78 (m, 3H), 7.14-7.21 (m, 3H), $6.71(\mathrm{~d}, J=8.2 \mathrm{~Hz}, 2 \mathrm{H}), 5.59$ (br, 1H), $4.27(\mathrm{t}, J=6.8 \mathrm{~Hz}, 2 \mathrm{H})$, $3.36(\mathrm{t}, J=6.3 \mathrm{~Hz}, 2 \mathrm{H}), 3.12(\mathrm{t}, J=6.8 \mathrm{~Hz}, 2 \mathrm{H}), 2.60(\mathrm{t}, J=6.3 \mathrm{~Hz}, 2 \mathrm{H}) ;{ }^{13} \mathrm{C}-\mathrm{NMR} \delta: 172.3,155.8$, 136.1, 134.5, 132.9, 131.4, 131.1, 130.6, 127.6, 124.2, 116.4, 63.2, 34.5 , 31.4, 22.5; Anal. Calcd. for $\mathrm{C}_{17} \mathrm{H}_{17} \mathrm{Cl}_{2} \mathrm{NO}_{2} \mathrm{~S}: \mathrm{C}, 55.14 ; \mathrm{H}, 4.63$. Found: C, 55.10; H, 4.65.

3-Morpholin-4-ylpropionic acid 2-phenylsulfanyl-ethyl ester (6): Off-white oil; ${ }^{1} \mathrm{H}-\mathrm{NMR} \delta$ : $7.42-7.38$ (m, 2H), 7.28-7.34 (m, 2H), 7.25-7.22 (m, 1H), 4.27 (t, $J=6.8 \mathrm{~Hz}, 2 \mathrm{H}), 3.73-3.71(\mathrm{~m}, 4 \mathrm{H}), 3.15$ (t, $J=6.8 \mathrm{~Hz}, 2 \mathrm{H}), 2.71(\mathrm{t}, J=7.2 \mathrm{~Hz}, 2 \mathrm{H}), 2.38-2.46(\mathrm{~m}, 6 \mathrm{H}) ;{ }^{13} \mathrm{C}-\mathrm{NMR} \delta: 171.9,135.1,129.9,129.1$, 126.6, 66.5, 63.0, 53.6, 53.1, 32.4, 31.4; Anal. Calcd. for $\mathrm{C}_{15} \mathrm{H}_{21} \mathrm{NO}_{3} \mathrm{~S}: \mathrm{C}, 60.99$; H, 7.17. Found: C, $60.97 ; \mathrm{H}, 7.16$.

3-Diethylamino-propionic acid 2-phenylsulfanyl-ethyl ester (7): Off-white oil; ${ }^{1} \mathrm{H}-\mathrm{NMR} \delta$ : 7.13-7.28 $(\mathrm{m}, 5 \mathrm{H}), 4.46(\mathrm{t}, J=6.8 \mathrm{~Hz}, 2 \mathrm{H}), 3.74(\mathrm{t}, J=6.3 \mathrm{~Hz}, 2 \mathrm{H}), 3.12(\mathrm{t}, J=6.8 \mathrm{~Hz}, 2 \mathrm{H}), 2.78(\mathrm{t}, J=6.3 \mathrm{~Hz}$, $2 \mathrm{H}), 2.43(\mathrm{q}, J=6.2 \mathrm{~Hz}, 4 \mathrm{H}), 1.10(\mathrm{t}, J=6.2 \mathrm{~Hz}, 6 \mathrm{H}) ;{ }^{13} \mathrm{C}-\mathrm{NMR} \delta: 172.1,135.6,128.7,126.6,124.9$, 66.5, 48.6, 46.8, 53.1, 34.8, 16.7; Anal. Calcd. for $\mathrm{C}_{15} \mathrm{H}_{23} \mathrm{NO}_{2} \mathrm{~S}$ : C, 64.02; H, 8.24. Found: C, 64.04; $\mathrm{H}, 8.25$.

3-Piperidin-1-yl-propionic acid 2-phenylsulfanyl-ethyl ester (8): Off-white oil; ${ }^{1} \mathrm{H}-\mathrm{NMR} \delta$ : 7.75-7.85 $(\mathrm{m}, 3 \mathrm{H}), 7.43-7.51(\mathrm{~m}, 2 \mathrm{H}), 4.33(\mathrm{t}, J=6.8 \mathrm{~Hz}, 2 \mathrm{H}), 3.68(\mathrm{t}, J=7.2 \mathrm{~Hz}, 2 \mathrm{H}), 2.63(\mathrm{t}, J=6.8 \mathrm{~Hz}, 2 \mathrm{H})$, 2.44 (t, $J=7.2 \mathrm{~Hz}, 2 \mathrm{H}), 2.34$ (br, 10H); ${ }^{13} \mathrm{C}-\mathrm{NMR} \delta: 171.9,135.1,129.9,129.1,126.6,66.5,63.0,53.6$, 53.1, 32.4, 31.4, 29.8; Anal. Calcd. for $\mathrm{C}_{16} \mathrm{H}_{23} \mathrm{NO}_{2} \mathrm{~S}$ : C, 65.49; H, 7.90. Found: C, 65.51; H, 7.91.

3-Benzylaminopropionic acid 2-phenylsulfanyl-ethyl ester (9): Off-white oil; ${ }^{1} \mathrm{H}-\mathrm{NMR} \delta$ : 7.21-7.38 (m, $10 \mathrm{H}), 4.27(\mathrm{t}, J=6.8 \mathrm{~Hz}, 2 \mathrm{H}), 3.81(\mathrm{~s}, 2 \mathrm{H}), 3.16(\mathrm{t}, J=6.3 \mathrm{~Hz}, 2 \mathrm{H}), 2.90$ (t, $J=6.8 \mathrm{~Hz}, 2 \mathrm{H}), 2.52$ (t, $J=6.3 \mathrm{~Hz}, 2 \mathrm{H}) ;{ }^{13} \mathrm{C}-\mathrm{NMR} \delta: 172.5,140.1,135.1,130.0,129.1,128.5,128.0,127.0,126.6,62.8,53.8$, 44.4, 34.7, 32.4; Anal. Calcd. for $\mathrm{C}_{18} \mathrm{H}_{21} \mathrm{NO}_{2} \mathrm{~S}$ : C, 68.54; H, 6.71. Found: C, 68.53; H, 6.68.

3-Imidazol-1-yl-propionic acid 2-phenylsulfanyl-ethyl ester (10): Off-white oil; ${ }^{1} \mathrm{H}-\mathrm{NMR} \delta$ : 7.56 (s, 1H), 6.97-7.09 (m, 5H), $6.87(\mathrm{~d}, J=7.3 \mathrm{~Hz}, 2 \mathrm{H}), 4.36(\mathrm{t}, J=6.8 \mathrm{~Hz}, 2 \mathrm{H}), 3.96(\mathrm{t}, J=6.3 \mathrm{~Hz}, 2 \mathrm{H}), 3.16$ $(\mathrm{t}, J=6.8 \mathrm{~Hz}, 2 \mathrm{H}), 2.60(\mathrm{t}, J=6.3 \mathrm{~Hz}, 2 \mathrm{H}) ;{ }^{13} \mathrm{C}-\mathrm{NMR} \delta: 170.2,141.3,129.9,127.9 ., 126.7,125.2$, 124.8, 122.9, 65.6, 39.5, 32.4, 31.6; Anal. Calcd. for $\mathrm{C}_{14} \mathrm{H}_{16} \mathrm{~N}_{2} \mathrm{O}_{2} \mathrm{~S}$ : C, 60.85; H, 5.84. Found: C, 60.87; $\mathrm{H}, 5.82$.

3-(2-Aminophenylsulfanyl)propionic acid 2-phenylsulfanyl-ethyl ester (11): Off-white Oil; ${ }^{1} \mathrm{H}-\mathrm{NMR} \delta$ : 7.15-7.42 (m, $J=7.3 \mathrm{~Hz}, 7 \mathrm{H}), 6.67-6.75(\mathrm{~m}, 2 \mathrm{H}), 4.40(\mathrm{br}, 2 \mathrm{H}), 4.25(\mathrm{t}, J=6.8 \mathrm{~Hz}, 2 \mathrm{H}), 3.15$ (t, 
$J=6.3 \mathrm{~Hz}, 2 \mathrm{H}), 2.96(\mathrm{t}, J=6.8 \mathrm{~Hz}, 2 \mathrm{H}), 2.54(\mathrm{t}, J=6.3 \mathrm{~Hz}, 2 \mathrm{H}) ;{ }^{13} \mathrm{C}-\mathrm{NMR} \delta: 171.7,148.7,136.6$, $135.0,130.2,130.0,129.1,126.7,118.5,116.4,115.0,63.2,34.5,32.4,29.5$; Anal. Calcd. for $\mathrm{C}_{17} \mathrm{H}_{19} \mathrm{NO}_{2} \mathrm{~S}_{2}$ : C, 61.23; H, 5.74. Found: C, 61.25; H, 5.72.

3-(3-Ethyl-4-hydroxyphenylsulfanyl)propionic acid 2-phenylsulfanyl-ethyl ester (12): yellow oil; ${ }^{1} \mathrm{H}-$ NMR $\delta$ : 7.15-7.43 (m, 6H), 6.69-6.72 (m, $J=8.2 \mathrm{~Hz}, 2 \mathrm{H}), 5.56(\mathrm{br}, 1 \mathrm{H}), 4.27(\mathrm{t}, J=6.8 \mathrm{~Hz}, 2 \mathrm{H}), 3.15$ $(\mathrm{t}, J=6.3 \mathrm{~Hz}, 2 \mathrm{H}), 3.02(\mathrm{t}, J=6.8 \mathrm{~Hz}, 2 \mathrm{H}), 2.54-2.66(\mathrm{~m}, 4 \mathrm{H}), 1.24(\mathrm{t}, J=7.6 \mathrm{~Hz}, 3 \mathrm{H}) ;{ }^{13} \mathrm{C}-\mathrm{NMR} \delta$ : 172.1, 153.6, 135.0, 134.1, 131.7, 131.2, 130.0, 129.1, 126.7, 124.7, 115.9, 63.2, 34.5, 32.4, 31.1, 22.9, 13.9; Anal. Calcd. for $\mathrm{C}_{19} \mathrm{H}_{22} \mathrm{O}_{3} \mathrm{~S}_{2}$ : C, 62.95; H, 6.12. Found: C, 62.92; H, 6.14.

3-Phenylaminopropionic acid 2-(naphthalen-2-ylsulfanyl)-ethyl ester (13): yellow oil; ${ }^{1} \mathrm{H}-\mathrm{NMR} \delta$ : 7.73-7.81 (m, 4H), 7.42-7.52 (m, 4H), 7.04 (d, $J=8.1 \mathrm{~Hz}, 2 \mathrm{H}), 6.45$ (d, $J=8.1 \mathrm{~Hz}, 2 \mathrm{H}), 4.29$ (t, $J=6.7 \mathrm{~Hz}, 2 \mathrm{H}), 3.91(\mathrm{br}, 1 \mathrm{H}), 3.41(\mathrm{t}, J=6.3 \mathrm{~Hz}, 2 \mathrm{H}), 3.24(\mathrm{t}, J=6.7 \mathrm{~Hz}, 2 \mathrm{H}), 2.57$ (t, $J=6.3 \mathrm{~Hz}$, $2 \mathrm{H}) ;{ }^{13} \mathrm{C}-\mathrm{NMR} \delta$ : 172.0, 145.6, 132.9, 132.6, 131.7, 129.9, 128.2, 128.2, 127.9, 127.5, 127.2, 126.4, 126.2, 116.7, 112.8, 63.4, 39.5, 33.3, 32.1; Anal. Calcd. for $\mathrm{C}_{21} \mathrm{H}_{21} \mathrm{NO}_{2} \mathrm{~S}$ : C, 71.76; H, 6.02. Found: C, $71.79 ; \mathrm{H}, 6.00$.

3-p-Tolylaminopropionic acid 2-(naphthalen-2-ylsulfanyl)-ethyl ester (14): white solid, m.p. 75-77 ${ }^{\circ} \mathrm{C}$; ${ }^{1} \mathrm{H}-\mathrm{NMR} \delta$ : 7.76-7.83 (m, 4H), 7.49 (t, $\left.J=8.4 \mathrm{~Hz}, 3 \mathrm{H}\right), 7.01(\mathrm{~d}, J=8.1 \mathrm{~Hz}, 2 \mathrm{H}), 6.56(\mathrm{~d}, J=8.1 \mathrm{~Hz}$, 2H), 4.32 (q, $J=6.7 \mathrm{~Hz}, 2 \mathrm{H}), 3.90(\mathrm{br}, 1 \mathrm{H}), 3.41(\mathrm{t}, J=6.3 \mathrm{~Hz}, 2 \mathrm{H}), 3.25(\mathrm{~m}, J=6.7 \mathrm{~Hz}, 2 \mathrm{H}), 2.57$ (t, $J=6.3 \mathrm{~Hz}, 2 \mathrm{H}), 2.51(\mathrm{~s}, 3 \mathrm{H}) ;{ }^{13} \mathrm{C}-\mathrm{NMR} \delta: 172.2,145.2,133.7,132.4,132.0,129.8,128.7,128.2$, $128.1,127.7,127.2,127.0,126.7,126.0,113.4,62.9,39.8,33.9,32.4,20.4$; Anal. Calcd. for $\mathrm{C}_{22} \mathrm{H}_{23} \mathrm{NO}_{2} \mathrm{~S}: \mathrm{C}, 72.30 ; \mathrm{H}, 6.34$. Found: C, 72.32; H, 6.36.

3-(4-Methoxyphenylamino)propionic acid 2-(naphthalen-2-ylsulfanyl)-ethyl ester (15): Off-white oil; ${ }^{1} \mathrm{H}-\mathrm{NMR} \delta$ : 7.78-7.89 (m, 4H), 7.46-7.54 (m, 3H), $6.82(\mathrm{~d}, J=8.8 \mathrm{~Hz}, 2 \mathrm{H}), 6.63(\mathrm{~d}, J=8.8 \mathrm{~Hz}, 2 \mathrm{H})$, $4.35(\mathrm{t}, J=6.8 \mathrm{~Hz}, 2 \mathrm{H}), 3.82(\mathrm{br}, 1 \mathrm{H}), 3.75(\mathrm{~s}, 3 \mathrm{H}), 3.38(\mathrm{t}, J=6.3 \mathrm{~Hz}, 2 \mathrm{H}), 3.27(\mathrm{t}, J=6.3 \mathrm{~Hz}, 2 \mathrm{H})$, $2.58(\mathrm{t}, J=6.8 \mathrm{~Hz}, 2 \mathrm{H}) ;{ }^{13} \mathrm{C}-\mathrm{NMR} \delta: 172.2,152.6,141.7,133.8,132.5,132.0,128.7,128.0,127.9$, 127.7, 127.2, 126.7, 126.0, 115.1, 114.8, 63.1, 55.8, 40.6, 33.9, 32.3; Anal. Calcd. for $\mathrm{C}_{22} \mathrm{H}_{23} \mathrm{NO}_{3} \mathrm{~S}: \mathrm{C}$, 69.26; H, 6.08. Found: C, 69.29; H, 6.05.

3-Morpholin-4-yl-propionic acid 2-(naphthalen-2-ylsulfanyl)-ethyl ester (16): yellow oil; ${ }^{1} \mathrm{H}-\mathrm{NMR} \delta$ : 7.73-7.83 (m, 4H), 7.40-7.49 (m, 3H), $4.31(\mathrm{t}, J=6.8 \mathrm{~Hz}, 2 \mathrm{H}), 3.67(\mathrm{t}, J=4.6 \mathrm{~Hz}, 4 \mathrm{H}), 3.23$ (t, $J=6.8 \mathrm{~Hz}, 2 \mathrm{H}), 2.64(\mathrm{t}, J=7.3 \mathrm{~Hz}, 2 \mathrm{H}), 2.41-2.46(\mathrm{tt}, J=7.3 \mathrm{~Hz}, J=4.6 \mathrm{~Hz}, 6 \mathrm{H}) ;{ }^{13} \mathrm{C}-\mathrm{NMR} \delta: 171.0$, 135.1, 133.9, 128.3, 127.2, 126.5, 126.3, 126.1, 124.7, 66.5, 63.0, 53.6, 53.1, 32.4, 31.4; Anal. Calcd. for $\mathrm{C}_{19} \mathrm{H}_{23} \mathrm{NO}_{3} \mathrm{~S}$ : C, 66.06; H, 6.71. Found: C, 66.08; H, 6.73.

\section{Conclusions}

In conclusion, we have described herein the scope and limitations of the use of silica sulfuric acid ( $\mathrm{SSA}$ or $\mathrm{SiO}_{2}-\mathrm{SO}_{3} \mathrm{H}$ ) as a highly efficient catalyst for Michael additions of amines under solvent-free conditions. This process avoids the use of organic solvent to carry out the reaction, and the catalyst can 
be separated from the product very easily after the reaction. Handling of the catalyst is easy, and it can be used again.

\section{Acknowledgements}

This work was funded by the Natural Science Foundation of Zhejiang Province, P.R. China (Project Y407240) and key project of Lishui University.

\section{References}

1. Azizi, N.; Saidi, M.R. Novel and efficient method for the silylation of hydroxyl groups with hexamethyldisilazane (HMDS) under solvent-free and neutral conditions. Organometallics 2004, 23, 1457-1458.

2. Azizi, N.; Saidi, M.R. LiClO4 accelerated Michael addition of amines to $\alpha, \beta$ - unsaturated olefins under solvent-free conditions. Tetrahedron 2004, 60, 383-387.

3. Azizi, N.; Saidi, M.R. Lithium perchlorate-catalyzed three-component coupling: A facile and general method for the synthesis of $\alpha$-aminophosphonates under solvent-free condition. Eur. J. Org. Chem. 2003, 4630-4633.

4. Azizi, N.; Saidi, M.R. Highly chemoselective addition of amines to epoxides in water. Org. Lett. 2005, 7, 3649-3651.

5. Yang, L.; Xu, L.W.; Zhou, W.; Li, L.; Xia, C.G. Highly efficient aza-Michael reactions of aromatic amines and N-heterocycles catalyzed by a basic ionic liquid under solvent-free conditions. Tetrahedron Lett. 2006, 47, 7723-7726.

6. Krishna, P. R.; Sreeshailam, A.; Srinivas, R. Recent advances and applications in asymmetric aza-Michael addition chemistry. Tetrahedron 2009, 65, 9657-9672.

7. Bartoli, G.; Bosco, M.; Marcantoni, E.; Petrini, M.; Sambri, L.; Torregiani, E. Conjugate addition of amines to $\alpha, \beta$-enones promoted by $\mathrm{CeCl} 3 \cdot 7 \mathrm{H} 2 \mathrm{O}-\mathrm{NaI}$ system supported in silica gel. J. Org. Chem. 2001, 66, 9052-9055.

8. Srivastava, N.; Banik, B.K. Bismuth nitrate-catalyzed versatile Michael reactions. J. Org. Chem. 2003, 68, 2109-2114.

9. Varala, R.; Alam, M.M.; Adapa, S.R. Chemoselective michael type addition of aliphatic amines to $\alpha, \beta$-ethylenic compounds using bismuth triflate catalyst. Synlett 2003, 720-722.

10. Saidi, M.R.; Pourshojaei, Y.; Fezzeh, A. Highly efficient michael addition reaction of amines catalyzed by silica-supported aluminum chloride. Synthetic Commun. 2009, 39, 1109-1119.

11. Yadav, J.S.; Ramesh Reddy, A.; Gopal Rao, Y.; Narsaiah, A.V.; Reddy, B.V.S. Lanthanum trichloride $\left(\mathrm{LaCl}_{3}\right)$ : An efficient catalyst for conjugate addition of amines to electron-deficient olefins. Lett. Org. Chem. 2007, 4, 462-464.

12. Khan, A.T.; Parvin, T.; Gazi, S.; Choudhury, L.H. Bromodimethyl sulfoniumbromide-mediated Michael addition of amines to electron deficient alkenes. Tetrahedron Lett. 2007, 48, 3805-3808.

13. Mukherjee, C.; Misra, A.K. Aza-Michael addition of amines to activated alkenes catalyzed by silica supported perchloric acid under a solvent-free condition. Lett. Org. Chem. 2007, 4, 54-59.

14. Duan, Z.; Xuan, X.; Li, T.; Yang, C.; Wu, Y. Cerium(IV) ammonium nitrate (CAN) catalyzed 
aza-Michael addition of amines to $\alpha, \beta$-unsaturated electrophiles. Tetrahedron Lett. 2006, 47, 5433-5436.

15. Varala, R.; Sreelatha, N.; Adapa, S.R. Ceric ammonium nitrate catalyzed aza-Michael addition of aliphatic amines to $\alpha, \beta$-unsaturated carbonyl compounds and nitriles in water. Synlett 2006, 1549-1553.

16. Surendra, K.; Krishnaveni, N.S.; Sridhar, R.; Rao, K.R. b-Cyclodextrin promoted aza-Michael addition of amines to conjugated alkenes in water. Tetrahedron Lett. 2006, 47, 2125-2127.

17. Meshram, H.M.; Lakshindra, C.; Reddy, P.N.; Sadashiv, K.; Yadav, J.S. Zirconium(IV) chloride-mediated chemoselective conjugate addition of aliphatic amines to $\alpha, \beta$-ethylenic compounds. Synth. Commun. 2006, 36, 795-801.

18. Yadav, J.S.; Ramesh Reddy, A.; Gopal Rao, Y.; Narsaiah, A.V.; Subba Reddy, B.V. Samarium(III) triflate catalyzed conjugate addition of amines to electron-deficient alkenes. Synthesis 2007, 3447-3450.

19. Hashemi, M.M.; Eftekhari-Sis, B.; Abdollahifar, A.; Khalili, B. $\mathrm{ZrOCl}_{2} \cdot 8 \mathrm{H}_{2} \mathrm{O}$ on montmorillonite $\mathrm{K}_{10}$ accelerated conjugate addition of amines to a,b-unsaturated alkenes under solvent-free conditions. Tetrahedron 2006, 62, 672-677.

20. Bo, K.L.; Wu, Q.; Xue, Q.Q.; De, S.L.; Xian, F.L. N-methyl-imidazole as a promising catalyst for the aza-Michael addition reaction of N-heterocycles. Synthesis 2007, 2653-2659.

21. Kantam, M.L.; Neeraja, V.; Kavita, B.; Neelima, B.; Chaudhuri, M.K.; Hussain, S. Cu(acac) $)_{2}$ immobilized in ionic liquids: A recoverable and reusable catalytic system for aza-Michael reactions. Adv. Synth. Catal. 2005, 347, 763-766

22. Yeom, C.E.; Kim, M.J.; Kim, B.M.; Choudhury, L.H. 1,8-Diazabicyclo [5.4.0]undec-7-ene (DBU)-promoted efficient and versatile aza-Michael addition. Tetrahedron 2007, 63, 904-909.

23. Monfray, J.; Koskinen, A.M.P. Aza-Michael additons on a,b-unsaturated esters catalyzed by bismuth(III) triflate in conventional chemistry and under microwave irradiation. Lett. Org. Chem. 2006, 3, 324-327.

24. Vijender, M.; Kishore, P.; Satyanarayana, B. Cadmium chloride $\left(\mathrm{CdCl}_{2}\right)$ : An efficient catalyst for conjugate addition of amines to electron-poor alkenes. Synth. Commun. 2007, 37, 591-594.

25. Bhanushali, M.J.; Nandurkar, N.S.; Jagtap, S.R.; Bhanage, B.M. $\mathrm{Y}\left(\mathrm{NO}_{3}\right)_{3} \cdot 6 \mathrm{H}_{2} \mathrm{O}$ catalyzed aza-Michael addition of aromatic/hetero or aromatic amines under solvent-free conditions. Catal. Commun. 2008, 9, 1189-1195.

26. Reddy, K.R.; Kumar, N.S. Cellulose-supported copper(0) catalyst for aza-Michael addition. Synlett 2006, 2246-2250.

27. Pore, D.M.; Soudagar, M.S.; Desai, U.V. Potassium phosphate or silica sulfuric acid catalyzed conjugate addition of thiols to alpha,beta-unsaturated ketones at room temperature under solvent-free conditions. Tetrahedron Lett.2006, 47, 9325-9328.

28. Minakata, S.; Komatsu, M. Organic Reactions on Silica in Water. Chem. Rev. 2009, 109, 711-724.

29. Salehi, P.; Zolfigol, M.A.; Shirini, F.; Baghbanzadeh, M. silica sulfuric acid and silica chloride as efficient reagents for organic reactions. Curr. Org. Chem. 2006, 10, 2171-2189.

30. Shaabani, A.; Sarvary, A.; Rahmati, A.; Rezayan, A.H. ionic liquid/silica sulfuric acid promoted fast synthesis of a Biginelli-Like scaffold reaction. Lett. Org. Chem. 2007, 4, 68-71.

31. Li, J.T.; Dai, H.G.; Xu, W.Z.; Li, T.S. Michael addition of indole to $\alpha, \beta$-unsaturated ketones 
catalysed by silica sulfuric acid under ultrasonic irradiation. J. Chem. Res. 2006, 41-42.

32. Wu, H; Shen, Y; Fan, L.Y.; Wan, Y; Zhang, P; Chen, C.F.; Wang, W.X. Stereoselective synthesis of $\beta$-amino ketones via direct Mannich-type reaction catalyzed with silica sulfuric acid. Tetrahedron 2007, 63, 2404-2408.

33. Shirini, F.; Zolfigol, M.A.; Mohammadi, K. Silica sulfuric scid as a mild and efficient reagent for the acetylation of alcohols in solution and under solvent free conditions Bull. Korean Chem. Soc. 2004, 25, 325-327.

34. Modarresi, A.R.; Nasrollahzadeh, M.; Khamooshi. F. Solvent-free preparation of primary carbamates using silica sulfuric acid as an efficient reagent. ARKIVOC 2007, xvi, 238-245.

Sample Availability: Contact the authors.

(C) 2009 by the authors; licensee Molecular Diversity Preservation International, Basel, Switzerland. This article is an open-access article distributed under the terms and conditions of the Creative Commons Attribution license (http://creativecommons.org/licenses/by/3.0/). 